\title{
Building capacity for antiretroviral delivery in South Africa: A qualitative evaluation of the PALSA PLUS nurse training programme
}

\author{
J Stein*1, S Lewin²,3, L Fairall'4, P Mayers5,6, R English7 , A Bheekie8, \\ E Bateman ${ }^{9}$ and M Zwarenstein ${ }^{10,11}$
}

\begin{abstract}
Address: ${ }^{1}$ University of Cape Town Lung Institute, George St, Mowbray 7700, Cape Town, South Africa, ${ }^{2}$ Department of Public Health and Policy, London School of Hygiene and Tropical Medicine, UK, ${ }^{3}$ Health Systems Research Unit, Medical Research Council of South Africa, PO Box 19070 , Tygerberg 7505, Cape Town, South Africa, ${ }^{4}$ University of Cape Town Lung Institute, George St, Mowbray 7700, Cape Town, South Africa, ${ }^{5}$ School of Health and Rehabilitation Sciences, Faculty of Health Sciences, University of Cape Town, South Africa, 6 University of Cape Town Lung Institute, George St, Mowbray 7700, Cape Town, South Africa, ${ }^{7}$ University of Cape Town Lung Institute, George St, Mowbray 7700, Cape Town, South Africa, ${ }^{8}$ School of Pharmacy, University of the Western Cape, P/Bag X17, Bellville 7535, Cape Town, South Africa, ${ }^{9}$ Department of Medicine, University of Cape Town Lung Institute, George St, Mowbray, Cape Town, South Africa, ${ }^{10}$ Centre for Health Services Sciences, Sunnybrook Research Institute, Toronto, Ontario, Canada and ${ }^{11}$ Department of Health Policy, Management and Evaluation, University of Toronto, Toronto, Ontario, Canada
\end{abstract}

Email: J Stein* - jostein@telkomsa.net; S Lewin - simon.lewin@nokc.no; L Fairall - lara.fairall@uct.ac.za; P Mayers - Pat.Mayers@uct.ac.za; R English - renglish@uct.ac.za; A Bheekie - abheekie@uwc.ac.za; E Bateman - ebateman@uct.ac.za;

M Zwarenstein - merrick.zwarenstein@ices.on.ca

* Corresponding author

Published: 18 November 2008

BMC Health Services Research 2008, 8:240 doi:10.1 186/1472-6963-8-240
Received: 13 November 2007

Accepted: 18 November 2008

This article is available from: http://www.biomedcentral.com/1472-6963/8/240

(c) 2008 Stein et al; licensee BioMed Central Ltd.

This is an Open Access article distributed under the terms of the Creative Commons Attribution License (http://creativecommons.org/licenses/by/2.0), which permits unrestricted use, distribution, and reproduction in any medium, provided the original work is properly cited.

\begin{abstract}
Background: South Africa recently launched a national antiretroviral treatment programme. This has created an urgent need for nurse-training in antiretroviral treatment (ART) delivery. The PALSA PLUS programme provides guidelines and training for primary health care (PHC) nurses in the management of adult lung diseases and HIVIAIDS, including ART. A process evaluation was undertaken to document the training, explore perceptions regarding the value of the training, and compare the PALSA PLUS training approach (used at intervention sites) with the provincial training model. The evaluation was conducted alongside a randomized controlled trial measuring the effects of the PALSA PLUS nurse-training (Trial reference number ISRCTN24820584).

Methods: Qualitative methods were utilized, including participant observation of training sessions, focus group discussions and interviews. Data were analyzed thematically.

Results: Nurse uptake of PALSA PLUS training, with regard not only to ART specific components but also lung health, was high. The ongoing on-site training of all PHC nurses, as opposed to the once-off centralized training provided for ART nurses only at non-intervention clinics, enhanced nurses' experience of support for their work by allowing, not only for ongoing experiential learning, supervision and emotional support, but also for the ongoing managerial review of all those infrastructural and system-level changes required to facilitate health provider behaviour change and guideline implementation. The training of all PHC nurses in PALSA PLUS guideline use, as opposed to ART nurses only, was also perceived to better facilitate the integration of AIDS care within the clinic context.
\end{abstract}


Conclusion: PALSA PLUS training successfully engaged all PHC nurses in a comprehensive approach to a range of illnesses affecting both HIV positive and negative patients. PHC nursetraining for integrated systems-based interventions should be prioritized on the ART funding agenda. Training for individual provider behaviour change is nonetheless only one aspect of the ongoing system-wide interventions required to effect lasting improvements in patient care in the context of an over-burdened and under-resourced PHC system.

\section{Background}

In South Africa, where over 5 million people are HIV infected, and at least 900000 people are estimated to be eligible for antiretroviral treatment (ART), the roll-out of the ART programme thus far has been slow and insufficient [1]. Nonetheless, it is estimated that more than 300 000 people have now accessed ART in South Africa through the public sector [2]. The rapid expansion of the ART programme has refocused attention on the need for the provision of integrated primary health care. This is partly because HIV, with its diverse manifestations, demands it. It is also because without an integrated health systems approach, the South African ART programme, heavily-injected with donor funding and potentially the largest in the world, would become a mammoth vertical structure of little benefit to the overall health service [3-5]; which is fragile, overburdened and in urgent need of additional resources. The pool of human resources in particular is so low that a vertical ART programme would continue, as has already been seen, to drain experienced personnel from the wider health service. The most compelling reason for an integrated $\mathrm{PHC}$ approach is that PHC in South Africa is conducted, almost exclusively, by nurses. It is the only level of the public health care structure which reaches most South African people, and therefore the only way for the ART programme to reach all those who need it. In the face of one of the most extensive AIDS epidemics in the world and a critical shortage of health care workers, especially doctors [6-8], it is clear that non-physician models of health care delivery are necessary if the constantly expanding ART programme is to be successful and sustainable [9].

\section{ART roll-out in the Free State Province}

The Free State province of South Africa has a high prevalence of HIV infection, with $31.1 \%$ of antenatal attendees testing positive [10]. Demographic modeling suggests that 48392 of the Free States 391527 HIV-positive citizens have AIDS [11], and so are presently eligible for ART in line with the National AIDS Plan [12]. Roll-out of the Free State ART programme started in May 2004 and at the end of March 2005, only 1165 had commenced treatment [13]. These figures have since increased and 9320 patients had commenced treatment by July 2007 (Venessa Timmerman, personal communication, as per FSDoH data warehouse, 2007).
The Free State, like South Africa as a whole, is characterized by a lack of skilled health workers. This is illustrated by the vacancy rate in 2005 of $40.7 \%$ for health professional posts in the province - a figure substantially higher than the national average of 31.1\%. [14]. PHC nurses play a pivotal role in the ART programme $[15,16]$. The roles and responsibilities of PHC nurses in the Free State ART programme have already grown substantially since the time of this study. At that time, which co-incided with the introduction of the programme in selected sites, nurses were responsible for screening and diagnosing patients; and assessing and referring those who qualified for treatment (i.e. those people with a CD4 count $=200$ and/or AIDS as diagnosed by clinical staging) to the nearest doctor (usually at a hospital) for assessment. Thereafter patients were referred back to nurse-run clinics where nurses issued medication and conducted clinical monitoring. A doctor reviewed progress twice during the first three months, and thereafter six-monthly.

\section{The PALSA PLUS approach}

PALSA PLUS is a health systems-based approach to training for primary care providers which attempts to capitalize on the opportunity provided by the ART roll-out to ensure that training for the ART programme is also used to strengthen overall health service delivery. This is in response to concerns that global initiatives such as ' 3 by 5 ' [1] have emphasized increasing the number of patients on ART without giving sufficient consideration to impacts on the primary health system $[3,4]$.

PALSA PLUS is based on the PALSA (Practical Approach to Lung Health in South Africa) programme. PALSA has been shown in a randomized controlled trial (RCT) to be a promising model for improving quality of care and the control of priority respiratory diseases, without extra staff, in resource poor primary care settings [17]. PALSA PLUS was developed at the request of the Free State province following the successful implementation of PALSA.

The "+" in PALSA PLUS was added when the programme was adapted and reconceptualized to incorporate the management of HIV and AIDS, including ART. The inclusion in PALSA PLUS of HIV/AIDS management is based on the rationale that South Africa has the highest co-infection rate of HIV and TB in the world [18], and that TB is 
the leading cause of death among HIV infected South Africans [19]. It therefore made clinical sense to combine a lung health training programme with the additional training on ART now required. This approach was seen as having the added advantage of protecting against vertical ART programme dominance and retaining attention on the management of under-diagnosed and under-treated priority diseases, including those that are HIV-related, such as tuberculosis, and other illnesses, such as asthma $[5,17]$.

\section{The PALSA PLUS guideline and training programme}

The PALSA PLUS intervention included two main components: (1) a comprehensive set of algorithm-based syndromic guidelines for the PHC nurse clinical management of respiratory diseases and HIV/AIDS; and (2) a training programme to facilitate guideline implementation. While the PALSA PLUS guidelines were evidence-based, they were also tailored to existing national and provincial models of care and designed to be applicable in underresourced PHC clinical settings. The guidelines were designed to be as simple and self-explanatory as possible. The intervention nonetheless involved a training programme to facilitate guideline use, given that the impacts of passive guideline dissemination on practice are modest [20].

PALSA PLUS nurse-training was based on the principles of educational outreach - a strategy shown to be effective in changing private health provider behaviour and practice [21]. These principles include the provision of short, faceto-face, in-service, interactive training by a trusted outsider. In accordance with educational outreach strategy, both the guidelines themselves and the training programme emphasized specific key behaviour change messages (i.e., relevant evidence-based diagnostic or treatment messages) which were seen to be most strongly correlated with improved treatment outcomes $[22,23]$.

Using a 'cascade' training approach [24], nurse-trainers were trained in both the content of the guideline and learner-centered group facilitation in order to transfer relevant knowledge and skills to PHC nurses at clinic level. Basic counselling principles, emphasizing the importance of guiding the client in taking responsibility for all healthrelated decision-making, were also conveyed and practiced in role-plays focusing on common clinical encounters. The core development team for the guidelines and for the training of the nurse-trainers included three medical doctors specializing in TB and AIDS care and a training facilitator with extensive expertise and experience in the nursing sector. Nine nurse-trainers, with varying degrees of medical knowledge and prior nurse-training experience and already employed by the provincial Department of Health as TB and HIV co-coordinators, were trained in a five day intensive live-in course. This training was followed by three six-weekly support visits from the core guideline and training development team during the course of each nurse-trainer's provision of training to PHC nurses at clinics province-wide. Informal individual support contacts with nurse-trainers were also provided.

The on-site training of PHC clinic nurses by nurse-trainers was conducted in weekly two hour sessions over a period of three to four months. This is in accordance with evidence to the effect that the alternation of learning and practice is the preferential model for the integration, testing and application of new knowledge $[25,26]$.

In order to help ensure replicability across the province, the duration and quality of both the training for nursetrainers and the on-site training of nurses themselves, was determined wholly by the constraints, particularly with regard to human resources, of the provincial Department of Health.

\section{Intervention and research design}

The fifteen clinics chosen by the Free State Department of Health to be involved in the first phase of the provincial ART roll-out were randomized to receive either the PALSA PLUS guidelines and a one week provincial training course only (control sites, $\mathrm{n}=7$ ) or to receive the provincial training and PALSA PLUS guidelines as well as an additional PALSA PLUS training package (intervention sites, $\mathrm{n}$ $=8)($ Table 1$)$.

The provincial training was provided to selected nurses newly appointed to the ART treatment programme (socalled "ART nurses") at both intervention and control sites and was conducted prior to the initiation of PALSA PLUS training at intervention sites. It is important to note that only the one or two nurses designated as "ART nurses" in each PHC clinic attended this provincial training. Provincial training included an introduction to the PALSA PLUS guideline and all nurses were given a copy of the guideline

Table I: Interventions received by the control and intervention sites

\begin{tabular}{ll}
\hline Control sites $(\mathbf{n}=\mathbf{7})$ & Intervention sites $(\mathbf{n}=\mathbf{8})$ \\
\hline $\begin{array}{l}\text { Provincial training (ART nurses only) } \\
\text { Dissemination of PALSA PLUS guidelines to ART nurses only }\end{array}$ & $\begin{array}{l}\text { Provincial training (ART nurses only) } \\
\text { Dissemination of PALSA PLUS guidelines and PALSA PLUS educational outreach } \\
\text { training to all clinic nurses }\end{array}$ \\
\hline
\end{tabular}


in their resource packs. Nurses were also provided with a structured opportunity to practice using the guidelines in role-plays. In all other respects, the provincial training may be described as essentially didactic in style, involving unidirectional presentations and passive dissemination of information and resource materials.

Thereafter, eight intervention sites received additional onsite PALSA PLUS training. The current evaluation compares and contrasts clinics which received the provincial training only with those that also received on-site, interactive training in the use of the PALSA PLUS guidelines. Table 2 highlights the key differences between the provincial and PALSA PLUS training approaches.

\section{Research Aim and Objectives}

The qualitative evaluation elaborated here explored the perceptions of those involved in the ART programme, especially PHC nurses, regarding the value of the PALSA PLUS training approach. This evaluation was grounded within a larger contextual exploration of nurse perceptions of the Free State ART roll-out, the results of which are discussed elsewhere [15]. The evaluation was conducted alongside an randomized trial measuring patient outcomes attendant on PALSA PLUS nurse-training, the results of which are forthcoming (Trial reference number ISRCTN24820584).

\section{Methods}

Qualitative data was collected in 2004 and 2005 using a variety of methods, including:

- Unstructured participant observation of the full five-day provincial training programme for 'ART nurses', i.e., those nurses selected to be involved in ART provision;

- Unstructured participant observation of the full five-day PALSA PLUS training of nurse-trainers and of all followup support workshops for nurse-trainers;

- Focus group discussions $(n=3)$ with all nine nursetrainers during the course of implementation of the training;
- Participant observation ( $\mathrm{n}=3$ sessions) of on-site PALSA PLUS nurse-training;

- Semi-structured interviews $(n=14)$ with four doctors in charge of district-level HIV clinics and ten nurses at two intervention and two control clinics.

Participant observation of provincial and PALSA PLUS training allowed for comparison of the training approaches adopted and nurses' responses to these. Both interviews and focus group discussions focused on respondents' perceptions of the value of the training they had received and their remaining training and support needs.

All participant observation and interview data were collected in English which was the language in which training was conducted. All data were tape-recorded, transcribed verbatim and analysed thematically. For each key analytic theme, data extracts were identified on the basis of being representative and/or interesting illustrations of an emerging issue [27]. All negative instances of the findings were discussed and accounted for.

The reliability and validity of the data were enhanced through iterative data collection, the use of a multimethod design and the ongoing discussion of findings within the research team for scrutiny and feedback $[28,29]$. Purposive sampling ensured that those clinics chosen for detailed study included both urban and rural sites with varying degrees of human and financial resources [30].

The study on which this paper is based has full ethical approval from the Research Ethics Committee of the Health Sciences Faculty of the University of Cape Town (IRB 00001938; REF 098/2004). The study was carried out in accordance with the criteria of the Declaration of Helsinki. Informed consent was obtained in writing from all participants and all information that might allow individuals to be identified has been deleted so as to protect their anonymity.

Table 2: Comparison of provincial and PALSA PLUS training approaches

\begin{tabular}{|c|c|}
\hline Provincial training approach & PALSA PLUS training approach \\
\hline Centralized by district & Decentralized (on-site at individual clinics) \\
\hline Doctors and nurses trained together & Training designed specifically for nurses \\
\hline Attendance by ART nurses only & Attendance by all nurses in each intervention clinic \\
\hline $\begin{array}{l}\text { Conference-type format: Expert presentations (primarily didactic and } \\
\text { uni-directional) by multiple specialists }\end{array}$ & $\begin{array}{l}\text { Educational outreach (facilitative, interactive) by trained group } \\
\text { facilitators }\end{array}$ \\
\hline Predominantly ART-specific AIDS care & Lung health (including TB), HIVIAIDS (including ART) care \\
\hline Multiple materials, including the PALSA PLUS guideline & PALSA PLUS guideline (and support materials) only \\
\hline
\end{tabular}




\section{Results}

By addressing the question: 'What extra value did the PALSA PLUS training add?', we hope to shed light on those specific aspects of the PALSA PLUS training which may have contributed to the more effective implementation and subsequent use of the treatment guidelines in intervention clinics. We argue below that the added value of PALSA PLUS training included the use of an educational outreach training approach which facilitated interactive learning, thereby allowing for the integration of learning and practice and the provision of both emotional and operational support. By "operational" support we refer to the active facilitation of the management support required to effect all those infrastructural changes required to make implementation of new diagnostic and treatment options, as outlined in the guidelines, feasible and practical. In addition, the involvement of all clinic nurses (rather than ART nurses only) in the training appeared to facilitate the horizontal system-wide integration of HIV/AIDS care.

\section{Training as facilitated interactive learning}

Nurses who had attended provincial training prior to PALSA PLUS training (i.e. ART nurses only) argued that although PALSA PLUS training covered much of the same material as the provincial training, it was more interactive and felt more like "training" and less like "an information session":

"I went to the provincial training before we started PALSA PLUS. Ja [yes], uhm, it sounded OK but it was, to me, uhm, more superficial. ...I found when you wanted to ask some questions, you felt you needed a session for us at clinic level. ... It [provincial training] was a high quality lecture, it was sort of an information session. And when I went to PALSA PLUS then I felt, "Now, we are being trained." (ART nurse 1, intervention site).

It was suggested that the PALSA PLUS training, which was conducted by HIV/AIDS and TB co-coordinators and designed specifically for nurses at clinic level, was less intimidating, more appropriate, and better attuned to the realities of primary care level treatment and care:

"I felt intimidated [at the provincial training]. When you are a primary care nurse and you come with actual problems at grassroots and when you pose such questions among high-ranking academics - because we had people from universities and doctors from hospitals lecturing - so now, we are talking another language. " (ART nurse 7, intervention site).

Nurses also valued the way in which the transfer of counselling skills was built into the PALSA PLUS nurse-training model and were keen to model the facilitative communi- cation skills demonstrated by nurse-trainers in the course of their own patient encounters. In the following data extract, the nurse explains that, unlike the provincial training, the PALSA PLUS training demonstrated a more patient-centered approach to the promotion of drugadherence:

"The modules from province is like a lecture. How can I give a sick person a lecture? A frustrated, miserable person a lecture? We were going up too high above the patient, when it must be done more practically. And the patient should be more involved." (ART nurse 6, intervention site).

\section{Integrating learning and practice}

Participants saw the PALSA PLUS on-site training approach, spread out over three to four months, as facilitating the gradual integration of new clinical information and skills. The integration of training and practical clinical work also allowed for ongoing supervisory feedback from nurse-trainers. This was seen to increase both the usefulness and the uptake of the training:

"We had time to go and revise... It was one chapter a week and from one Tuesday to the next Tuesday you've seen many TB cases, you know, you've actually applied what you learnt. So it was actually better, the PALSA PLUS. " (ART nurse 7 , intervention site).

"It's more relevant here [on-site] than outside because you can put it into practice. Whereas with off-site training, there's no evaluation of whether we are on the right track." (ART nurse 1, intervention site).

Participants nonetheless argued that their concentration and/or attendance at on-site training had suffered because of overlapping work-related commitments, especially the pressure of patients awaiting consultations. By comparison, centralized off-site training was seen to allow for more dedicated training time (i.e. a one-week block as compared to two-hour sessions over three to four months). Despite acknowledging the advantages of onsite training, some nurses therefore expressed a preference for training programmes which took them out of a stressful work environment. However, this disadvantage of onsite outreach training - the pressure experienced by nurses with patients awaiting their attention - is the flipside of what makes on-site training potentially more effective, through its immediate and direct link with 'real-life' patient service provision.

\section{Ongoing on-site training provides emotional support}

Nurses' need for emotional support was raised frequently. In their interviews, all nurses emphasized the high emotional toll of their ever-increasing workload with HIV pos- 
itive patients who, at this early stage of the roll-out, are generally very ill:

"If you are a human being, you will be touched [by the work with HIV positive patients]. It will work on your mind. You need to be strong and also develop skills to cope. Although, even so, you can't cope with everything." (ART nurse 6, intervention clinic)

Respondents noted that the ongoing engagement over time between PALSA PLUS nurse-trainers and clinic nurses provided them with much-needed support:

"I found them [PALSA PLUS trainers] accepting us. ... They try to reassure us, 'No, we are there, whatever the trouble, you must phone me"'. (Generalist nurse 3, intervention site).

By comparison, respondents argued that little formal provision had been made at provincial level for providing support to nurses involved in the initiation of the ART roll-out. Inadequate support from clinic-level managers (referred to below as 'supervisors') was also commonly reported:

"Our supervisors don't support us. They never come and ask, 'How are you, Can I help you?"' (ART nurse 2, intervention site).

Nurse-trainers also identified the supportive aspects of the PALSA PLUS training as one of its key successes:

"No, the responses [to the training] are very, very good. They have a feeling of support". (Nurse-trainer 2).

The ongoing nature of the training over time was, in and of itself, a source of reassurance and support:

"The trainers are really good. We ask them a question, and if they don't know, then the next week, they will come back with an answer." (ART nurse 2, intervention site).

Our observations of clinic training showed that the nursetrainers' roles expanded from training in guideline use alone to include both the provision of emotional support and ART programme facilitation. For example, some nurse-trainers involved themselves in supportive systemlevel interventions, including liaising and negotiating for required operational changes with managers at various levels of the system. Some nurse-trainers also became actively involved in 'head-hunting' staff for vacant clinic ART posts and ensuring adequate ART supplies. This was possible because, as provincial-level HIV and TB co-coordinators, trainers were in a position to effect such interventions. The incorporation of prayer into nurse-training sessions, as a means of accessing spiritual reserves for emotional support, is an example of PALSA PLUS nursetrainers incorporation of their own culturally appropriate support activities for nurses working at the frontlines of the epidemic [15]. The practical support and ongoing engagement on the part of trainers was especially appreciated by clinic nurses and may go a long way to explain the effects of the PALSA PLUS training and any differences in successful uptake of the guidelines between the intervention and control clinics.

\section{Horizontal system-wide integration of comprehensive HIVI AIDS care}

Nurses all agreed that specialized ART nurses are needed within the PHC system for the vertical management, monitoring and counselling of ART patients. This, they suggested, would help to ensure treatment continuity and a stronger patient-provider relationship. However, nurses also noted consistently that all PHC nurses needed training in ART provision to better manage ART patients suffering from opportunistic infections or drug side-effects. Likewise, it was suggested that ART nurses needed to locate ART provision within a more comprehensive approach to AIDS care.

It is interesting to note that the elaboration of non-ART related AIDS care within PALSA PLUS was often especially valued by nurses who received the PALSA PLUS training. By comparison, nurses at control sites expressed a need for additional training, in both ART and non-ART related aspects of AIDS care, especially TB care:

"The guidelines are simple and straightforward. But you do have to be trained to understand them, especially if you don't have PHC training, and especially if you don't know $T B$, which can be tricky if you [the patient] have HIV." (ART nurse 4, control site).

As mentioned previously, at control sites only those nurses designated as 'ART' nurses attended the provincial ART training and received their own copies of the PALSA PLUS guidelines. However, some of the PHC, or generalist, nurses at control sites made copies of their ART nurse colleagues' guidelines or specifically requested their own copies from provincial management. The guidelines were therefore widely disseminated - in the first year the document was printed three times to keep up with demand.

A PHC nurse at a control clinic (who had photocopied her ART colleague's PALSA PLUS guidelines) pointed out that the guidelines were especially valuable because they did not apply to ART provision or AIDS-defining conditions only, but to a wide range of conditions potentially exacerbated by HIV/AIDS: 
"The guidelines are like steps to follow. With regard to chest problems which are not responsive to antibiotics, it's guiding me properly - what steps to follow. Ja [yes], and it's not only that. There are so many conditions that they have put in." (Generalist nurse 9, control site).

By involving all clinic sisters in the training, PALSA PLUS presupposes that the treatment of ART patients should not be unnecessarily verticalized within the primary care context. In contrast, nurses at control sites suggested that, in the absence of training for PHC nurses, HIV/AIDS care as a whole had become specialized:

"It is best if everyone gets the training [in ART]. We are encountering problems. It's as if ART is a speciality but these patients are everywhere, at [the PHC side] as well. $H I V, A R V, T B$ : they go hand-in-hand." (ART nurse 10, control site).

"The ART nurse shouldn't have to treat patients for minor ailments just because they are [HIV] positive. The ART side is full. If they [PHC nurses] could have undergone training they could have known how to treat an HIV positive patient..." (PHC nurse 9, control site).

PHC nurses at control clinics felt particularly strongly about their need for training in AIDS care, including ART provision. They argued that they needed to be better equipped to treat the PHC needs of AIDS patients and so refer only ART-related complications to ART nurses. It was pointed out that a vertical HIV/AIDS programme was not feasible as the numbers of patients on ART was increasing and burn-out amongst ART nurse was inevitable:

"Everything concerning HIV happens at the ARV site. But they [ART nurses] need relief. ... The nurses become exhausted. They become depressed. "(PHC nurse 9, control site.)

"At the end of the day, 90\% of our patients [clinic-wide] are probably HIV positive." (PHC nurse 8, intervention site).

\section{Incorporating counselling skills into the PALSA PLUS model}

Skills development in counselling, including adherence counselling, was incorporated into training and support workshops for nurse-trainers. However, both participant observation and interview data suggest that additional training may be required if nurse-trainers are to be effectively empowered to convey practical counselling skills in a consistent and appropriate way to clinic-based nurses. Participant observation of on-site training showed that nurse-trainers were quick to adopt a model of PHC nursing as holistic care-giving. However, many had difficulty in conveying the practical skills required for practicing a more patient-centered and less technical model of patient care. Interview data also suggest that many nurses, including ART nurses, continue to have a limited understanding of their counselling role. They tend to see this as the provision of advice regarding drug adherence and other health-related behaviours rather than as patient empowerment for informed decision making:

"Some of them [some patients]...You give advice, they jump and do it. But the others are so difficult." (ART nurse 2, intervention site)

In this extract, the nurse conceives of counselling as 'advice' that must be complied with, rather than as a process of supporting the patient's expressed needs. Some PHC nurses also reported that they insist on HIV testing prior to treating the presenting condition of patients in order to ensure that patients with potentially AIDS-related symptoms 'agree' to VCT (voluntary testing and counselling):

"Sometimes, if you give them treatment, they'll not come back [for VCT]. ...I sit down and explain and explain until I win and then I go and hand them into the VCT counsellor's hands. I'm saying, 'See this lady and send her back to me so that she can have an appointment'. Because sometimes if you give them treatment, they'll not come back [for VCT]." (PHC nurse 8, intervention site).

Likewise, in order to achieve condom use or drug compliance, nurses stated that they resorted to a variety of persuasive strategies that are, arguably, at odds with a counselling role:

"I threaten them. 'I'm going to go to the police and tell them you're not using a condom and that you are killing people'..." (ART nurse 7, intervention site).

Despite expressing these somewhat prescriptive and seemingly punitive views, there was nonetheless also a strong shared sense between nurse-trainers and nurses of their role as caring and compassionate community members committed to helping those with HIV/AIDS. This provided a framework for increased investment in counselling training:

"I think emotional support and counselling must be, actually, in the [PALSA PLUS] guidelines because, after all, especially with these HIV people, they need that." (PHC nurse 8 , intervention site).

The PALSA PLUS guidelines and training were designed principally to facilitate evidence-based syndromic care and cannot substitute for skills-training in counselling. 
However, as a result of these research findings, key messages regarding counselling were subsequently incorporated into an updated version of the guidelines and training. This was on the grounds that nurses in the Free State saw the PALSA PLUS guideline as the anchor of the ART programme. This was especially so given that, in the absence of any other standardized national or provincial guidelines at the time, the PALSA PLUS guideline functioned as a very powerful and practical manifestation of the Free State's ART programme.

\section{Discussion}

The growing burden of HIV/AIDS in sub-Saharan Africa, and the urgent need to rollout treatment programmes, requires innovative approaches to building capacity for ART delivery and integrating comprehensive HIV/AIDS care within primary care. As ART becomes available more widely in many low and middle income country settings, it is becoming increasingly clear that human resources for health are the major constraint to ensuring that all those who need ART receive it, as part of a comprehensive package of care [31,32]. Little attention has yet been paid to how best to build capacity for ART rollout in underresourced settings without depleting other components of the health service of scarce human resources. The PALSA PLUS intervention begins to address this by training all nurses - the largest cadre of health care professionals in South Africa and many other African countries - in the clinical management of HIV/AIDS and respiratory diseases.

The PALSA PLUS model of clinic-based educational outreach targeting all professional nursing staff is a significant departure from current training approaches within the Free State health services and South Africa at large, as well as many other low and middle income countries. These training programmes tend to rely on either distance education or intensive off-site training with limited staff coverage [33]. This research indicates that clinic-based educational outreach can result not only in training coverage for a higher number of health workers but may also contribute to PHC integration by providing training to all PHC nurses, thereby facilitating horizontal rather than vertical HIV/AIDS care. PALSA PLUS may therefore contribute to much needed efforts to integrate the treatment of HIV/AIDS into the public PHC system [32].

An additional advantage of the PALSA PLUS training approach is its duration over time, allowing the provision of initial supervision to nurses newly involved in ART delivery. Diagnostic and treatment difficulties can be brought back to the nurse-trainers' attention and learning can be immediately integrated and translated into practice. Regular contact between trainers and trainees is also advantageous in that it allows training to provide much needed emotional and psychological support for nurses bearing the brunt of the AIDS epidemic. This has been shown to be an important need in this and other settings $[15,34,35]$. The choice of district-based HIV/AIDS and TB co-coordinators as nurse-trainers placed these trainers in an especially strong position to bring emerging issues to the attention of district managers, as well as to engage both clinic and district-level management in resolving operational bottlenecks in the health system. In doing so, nurse-trainers were able to provide an additional level of support to clinic nurses.

Our findings indicate that contextual factors at play at this moment in the history of the Free State province's healthservices ART roll-out may have resulted in a higher uptake of guidelines in control sites than can usually be expected of guidelines which are passively disseminated. These contextual factors include: (a) enthusiasm for the newly commenced ART roll-out and (b) the dearth of any other provincial guidelines regarding or including ART. Factors such as these may have made nurse enthusiasm for the guidelines as high in control sites (where guidelines were disseminated to ART nurses only) as in intervention sites (at which not just dissemination but training in guideline use for all nurses occurred). Any findings indicating improved treatment outcomes resulting from more effective guideline implementation at intervention clinics should arguably, therefore, be attributed in large part to the effectiveness of the training in facilitating guideline implementation, rather than simply to the relative failure of nurses at control clinics to engage enthusiastically with the guidelines simply because they were passively disseminated.

The PALSA PLUS training was thus perceived as going a long way in promoting, not just behaviour change at an individual health-provider level, but also a variety of broader system-level changes required to facilitate such individual behaviour change. A number of urgent health system challenges nonetheless remain which nurse-training alone cannot hope to address. Firstly, ART provision is more likely to be effective within a health system framework which consistently provides not only ART, but also a comprehensive package of PHC services ensuring prophylactic and curative treatment for opportunistic infections. Secondly, while a nurse-driven ART programme may help to address the problems resulting from an acute shortage of doctors, the fact remains that nurses at clinic level are also over-stretched, and that many PHC clinics are chronically under-staffed. This problem was expressed cogently by one of our respondents, "I think that the biggest problem is going to be human resources at the clinic. I mean, we are going to run out of staff. I just see the ARV roll-out just getting bigger and bigger until a point. ... It's just going to become a huge problem. " (Dr 1, Bloemfontein National Hos- 
pital). These are challenges not only for the Free State province of South Africa but also for health systems in other countries in sub-Saharan Africa $[8,32,36]$. Most of these countries are experiencing both critical absolute shortages in health care providers and poor distribution of providers within countries, exacerbated by deficiencies in skill mixes and poor physical and managerial infrastructures [8]. Addressing these deficits is key to improving health in Africa and reaching the Millennium Development Goals [37,38].

Findings suggest that training may have been insufficient to effect a deeper understanding of PHC providers' counseling role and that much more work needs to be undertaken in this area. Although counselling skills training was incorporated into the training intervention at the time of study, this was necessarily to a limited degree given the constraints on the programme. As a result of the findings of this study, relevant messages regarding the need for patient empowerment in decision-making have been integrated increasingly into the key messages elaborated in the guideline. The fact remains that effecting measurable improvements in those skills required to genuinely empower patients in decision-making may not be a realistic objective of the PALSA PLUS training intervention for some time. The PALSA PLUS programme necessarily prioritises its training objectives in order to achieve the best possible improvements in clinical treatment outcomes. What the programme currently aims to convey successfully is therefore not so much all necessary practical counseling skills per se, as an increasing conceptual appreciation of the rights of patients and of the roles of healthcare providers in helping patients towards autonomous decision-making on the basis of a neutral elaboration of their choices [39].

This evaluation of the PALSA PLUS training has several limitations. Firstly, its findings may not necessarily be generalisable to other settings in South Africa or beyond. This, however, is true for any programme evaluation, in so far as 'programmes differ from place to place because places differ' [40]. It nonetheless seems likely that the key aspects of the PALSA PLUS training programme identified by participants and outlined here are necessary if not sufficient to ensuring health practitioner behaviour change. Another significant limitation was that our study was limited to nurse perceptions and did not attempt to observe differences in nursing practices following training. The real effects of the PALSA PLUS intervention on nursing practices is considered by the RCT, the findings of which will be published separately. This process evaluation nonetheless shows that on-site training based on a 'cascade' model; drawing on the principles of educational outreach and interactive learning; and providing ongoing support to healthcare providers, is valued highly by pri- mary care nurses as facilitative of their work. Of particular importance is ensuring that healthcare providers receive, not just the motivation, skills and knowledge to implement new diagnostic and treatment guidelines, but also the management support to effect all those system-level changes required to make guideline implementation feasible.

Finally, this evaluation did not address the sustainability of the training effects in the medium term. Ongoing efforts from district TB and HIV/AIDS co-coordinators and managers will be necessary to ensure that nurses continue to receive the support and ongoing training that they require in the context of an increasing demand for treatment within an already over-extended and underresourced healthcare system [41].

\section{Conclusion}

The incorporation of ART treatment into the PALSA guidelines and training appears to have provided the 'hook' with which to engage PHC nurses in a comprehensive approach to improving quality of care for a range of illnesses affecting both HIV positive and negative patients. The integration of AIDS care and ART provision into a comprehensive package of PHC is in line with strong calls from the South African AIDS research community to prioritise systems-based interventions on the ART funding agenda.

Nurses at both intervention and control sites valued the PALSA PLUS guidelines highly. The added benefits of the PALSA PLUS training were seen to include the training of all clinic nurses, thereby facilitating the clinic-wide integration of HIV/AIDS care and ART provision; the interactive learning approach; the provision of support; and the integration of learning and practice.

Despite these encouraging findings, there can be little doubt that nurse-training alone, no matter how well designed, cannot overcome the more fundamental problems facing publicly funded health systems in the Free State and many other African contexts. These include insufficient human resources and an increasing disease burden due to HIV and TB. As the roles and responsibilities of nurses are expanded, not only in ART provision but also in the overall delivery of care in the PHC system, adequate training and support to all those responsible for ensuring programme implementation must continue to be provided. This includes not only PHC nurses themselves, but all those involved in the planning and management of the programme at all levels of the healthcare system. Without this ongoing investment, we will not be able to protect what wealth we do have: the commitment and capacity of nurses to deliver comprehensive PHC, including ART. 


\section{Competing interests}

The authors declare that they have no competing interests.

\section{Authors' contributions}

JS participated in the coordination and design of the study, executed the fieldwork and data analysis and drafted the manuscript. SL and LF participated in the coordination and design of the study, the critical review of the study findings, and the drafting of the manuscript. MZ, LF and EB conceptualized and initiated the PALSA PLUS programme. LF, PM, RE and AB designed and implemented the PALSA PLUS training programme. All authors read, contributed to and approved the final manuscript.

\section{Acknowledgements}

Thanks to the Free State Department of Health, especially the nurse-trainers and Free State clinic nurses who participated in this research and without whose commitment to the provision of comprehensive health care for all South Africans, and perseverance against conceivably soul-destroying odds, this work would not be possible. Simon Lewin is supported by the Medical Research Council of South Africa. The study was funded by the International Development Research Centre, Canada. After acceptance of the research protocol, the funder played no further role in determining the course or output of the research.

\section{References}

I. World Health Organisation \& UNAIDS: Progress on global access to HIV antiretroviral therapy. An update on "3 by 5". Geneva: WHO; 2005

2. Schneider $H$, van Rensburg D, Coetzee D: Health systems and antiretroviral access. Key findings and policy recommendations. Round table conference report. South Africa: Centre for Health Systems Research and Development, University of the Free State; 2007.

3. Chopra M: ARV treatment and health systems: Avoiding the pitfalls. AIDS Bulletin 2005, I 4(I):3-6.

4. McCoy D: Expanding access to ART in sub-Saharan Africa: an advocacy agenda for health systems development and resource generation. Acta Academica 2006: I-16.

5. Van Rensburg H, Schneider H: Preface to Acta Academica. Perspectives on public sector antiretroviral access. Acta Academica 2006:i-xi.

6. Kober K, Van Damme W: Scaling up access to antiretroviral treatment in southern Africa: who will do the job? Lancet 2004, 364(9428): 103-107.

7. Van Damme W, Kober K, Kegels G: Scaling-up antiretrovira treatment in Southern African countries with human resource shortage: how will health systems adapt? Soc Sci Med 2008, 66(10):2108-2121.

8. World Health Organisation: The World Health Report 2006 Working Together for Health. Geneva: WHO; 2006.

9. Jaffar S, Govender T, Garrib A, Welz T, Grosskurth H, Smith PG, Whittle $\mathrm{H}$, Bennish ML: Antiretroviral treatment in resourcepoor settings: public health research priorities. Trop Med Int Health 2005, I0(4):295-299.

10. National Department of Health: National HIV and Syphilis Antenatal Sero-prevalence Survey in South Africa. Pretoria: National Department of Health; 2002.

II. Dorrington R, Johnson L, Bradshaw D, Daniel T-J: The Demographic impact of AIDS in South Africa: National and Provincial indicators for 2006. 2006 [http://www.hivan.org.za/admin/ documents/ASSA2003Indicators.pdf]. Cape Town: UCT Centre for Actuarial Research, South Africa Medical Research Council and the Actuarial Society of South Africa

12. Operational Plan for comprehensive HIV and AIDS care, management and treatment for South Africa 2003 [http:// www.info.gov.za/issues/hiv/careplan.htm]. Pretoria, South Africa: National Department of Health
13. University of Cape Town SAMRC, FSDoH: Implementation of the comprehensive care, management and treatment of HIV and AIDS 2005. First Quarter Report. 2005 [http:// www.fs.gov.za/]. Cape Town: Medical Research Council and University of Cape Town Lung Institute for the Free State Department of Health

14. Doherty J, Loveday M, Stewart R, Thomas L: Conference report: Implementing the comprehensive care and treatment programme for HIV and AIDS patients in the Free State: sharing experiences. University of the Free State, Bloemfontein, South Africa. 30 March - I April 2005. 2005 [http:// www.hst.org.za/uploads/files/arvconf rep.pdf]. Durban: Health Systems Trust

15. Stein J, Lewin S, Fairall L: Hope is the pillar of the universe: health-care providers' experiences of delivering anti-retroviral therapy in primary health-care clinics in the Free State province of South Africa. Soc Sci Med 2007, 64(4):954-964.

16. Van Rensburg H: The Free State's approach to implementing the Comprehensive Plan: notes by a participant outsider. Perspectives on public sector antiretroviral access. Acta Academica 2006:44-93.

17. Fairall LR, Zwarenstein M, Bateman ED, Bachmann M, Lombard C, Majara BP, Joubert G, English RG, Bheekie A, van Rensburg D, et al: Effect of educational outreach to nurses on tuberculosis case detection and primary care of respiratory illness: pragmatic cluster randomised controlled trial. BMJ 2005, 33 I (75 I 9): $750-754$.

18. Corbett EL, Watt CJ, Walker N, Maher D, Williams BG, Raviglione $M C$, Dye $C$ : The growing burden of tuberculosis: global trends and interactions with the HIV epidemic. Arch Intern Med 2003, 163(9): 1009-102I.

19. Bradshaw D, Nannan N, Groenewald P, Joubert J, Laubscher R, Nojilana B, Norman R, Pieterse D, Schneider M: Provincial mortality in South Africa, 2000-priority-setting for now and a benchmark for the future. S Afr Med J 2005, 95(7):496-503.

20. Grimshaw JM, Thomas RE, MacLennan G, Fraser C, Ramsay CR, Vale L, Whitty P, Eccles MP, Matowe L, Shirran L, et al:: Effectiveness and efficiency of guideline dissemination and implementation strategies. Health Technol Assess 2004, 8(6):iii-iv. I-72

21. O'Brien MA, Rogers S, Jamtvedt G, Oxman AD, Odgaard-Jensen J, Kristoffersen DT, Forsetlund L, Bainbridge D, Freemantle N, Davis $\mathrm{DA}$, et al.: Educational outreach visits: effects on professional practice and health care outcomes. Cochrane Database Syst Rev 2007:CD000409.

22. Boissel IP, Haugh M, Fardeheb M, Nony P, Gueyffier F, Strang N: How should therapeutic information be transferred to users? Fundam Clin Pharmacol 2003, I 7(4):495-503.

23. Shaw B, Cheater F, Baker R, Gillies C, Hearnshaw H, Flottorp S, Robertson N: Tailored interventions to overcome identified barriers to change: effects on professional practice and health care outcomes. Cochrane Database Syst Rev 2005:CD005470.

24. Moys A, Mullick S, McCoy D, Beksinska M: Training clinic sisters. Lessons learnt based on experience of the national STI initiative. 2002 [http://www.hst.org.za/uploads/files/train nurse.pdf]. Durban: Reproductive Health Research Unit (RHRU) and Initiative for Sub-District Support (ISDS)

25. Bheekie A, Buskens I, Allen S, English R, Mayers P, Fairall L, Majara B, Bateman ED, Zwarenstein M, Bachmann M: The Practical Approach to Lung Health in South Africa (PALSA) intervention: respiratory guideline implementation for nurse trainers. Int Nurs Rev 2006, 53(4):26I-268.

26. Grol R: Implementing guidelines in general practice care. Qual Health Care 1992, I(3): 184-191.

27. Silverman D: Interpreting data. Methods for analyzing talk, text and interaction London: Sage; $200 \mathrm{I}$.

28. Marshall C, Rossman G: Designing qualitative research London: Sage; 1989.

29. Patton M: Qualitative research and evaluation methods Thousand Oaks: Sage; 2002.

30. Green J, Thorogood N: Qualitative methods for health research London: Sage; 2004.

31. Marchal B, De Brouwere V, Kegels G: Viewpoint: HIVIAIDS and the health workforce crisis: what are the next steps? Trop Med Int Health 2005, 10(4):300-304.

32. Schneider H, Blaauw D, Gilson L, Chabikuli N, Goudge J: Health systems and access to antiretroviral drugs for HIV in Southern 
Africa: service delivery and human resources challenges. Reprod Health Matters 2006, 14(27): 12-23.

33. Steyn F, van Rensberg H, Engelbrecht M: Human resources for ART in the Free State public Health Sector: recording achievements, identifying challenges. Perspectives on public sector antiretroviral access. Acta Academica 2006:94-137.

34. Shishana O, Hall E, Maluleke K, Stoker D, Schwabe C, Colvin M, Chauveau J, Botha C, Gumede T, Fomundam H, et al.: The impact of HIVIAIDS on the health sector. National survey of health personnel, ambulatory and hospitalised patients and health facilities, 2002. Pretoria: Human Sciences Research Council, Medunsa and Medical Research Council of South Africa; 2003.

35. Smit R: HIVIAIDS and the workplace: perceptions of nurses in a public hospital in South Africa. J Adv Nurs 2005, 5 I ( I ):22-29.

36. Joint Learning Initiative: Human resources for health: Overcoming the crisis. 2004 [http://www.hrhresourcecenter.org/node/3l]. Boston, MA: Joint Learning Initiative for Health

37. Lewin S, Lavis JN, Oxman AD, Bastias G, Chopra M, Ciapponi A, Flottorp S, Marti SG, Pantoja T, Rada G, et al.: Supporting the delivery of cost-effective interventions in primary health-care systems in low-income and middle-income countries: an overview of systematic reviews. Lancet 2008, 372(9642):928-939.

38. Sanders DM, Todd C, Chopra M: Confronting Africa's health crisis: more of the same will not be enough. BMJ 2005, 33 I (75 I 9): $755-758$.

39. Stein J, Steinberg M, Allwood C, Karstead A, Brouard P: Nursecounsellors' perceptions regarding HIVIAIDS. Counselling objectives at Baragwanath hospital. In The Impacts of AIDS: Psychological and social aspects of HIV Infection Edited by: Sherr L, Catalan J, Hedge B. Amsterdam, The Netherlands: Harwood Academic Publishers; 1997:191-198.

40. Edwards W, Guttentag M, Snapper K: A decision-theoretic approach to evaluation research. In Handbook of Evaluation Research Volume I. Edited by: Struening E, Guttentag M. Beverly Hills, CA: Sage; 1975.

41. Karaolis N, Jackson D, Ashworth A, Sanders D, Sogaula N, McCoy D, Chopra M, Schofield C: WHO guidelines for severe malnutrition: are they feasible in rural African hospitals? Arch Dis Child 2007, 92(3): 198-204.

\section{Pre-publication history}

The pre-publication history for this paper can be accessed here:

http://www.biomedcentral.com/1472-6963/8/240/pre

pub
Publish with Bio Med Central and every scientist can read your work free of charge

"BioMed Central will be the most significant development for disseminating the results of biomedical research in our lifetime. "

Sir Paul Nurse, Cancer Research UK

Your research papers will be:

- available free of charge to the entire biomedical community

- peer reviewed and published immediately upon acceptance

- cited in PubMed and archived on PubMed Central

- yours - you keep the copyright
BioMedcentral 